Pierre Hausfater

Bruno Megarbane

Sandrine Dautheville

Anabella Patzak

Marc Andronikof

Aline Santin

Stéphanie André

Ludovic Korchia

Nabila Terbaoui

Gérald Kierzek

Benoît Doumenc

Christophe Leroy

Bruno Riou

\section{Erratum to: Prognostic factors in non-exertional heatstroke}

Published online: 21 November 2009

(C) Copyright jointly hold by Springer and ESICM 2009

The online version of the original article can be found under doi:10.1007/s00134-009-1694-y.

P. Hausfater · B. Riou

Emergency Department, Centre Hospitalo-Universitaire (CHU)

Pitié-Salpêtrière, Assistance-Publique Hôpitaux de Paris,

UPMC Univ Paris 06, Paris, France

B. Megarbane

Critical Care Department, Centre Hospitalo-Universitaire (CHU)

Lariboisière, Assistance-Publique Hôpitaux de Paris, Paris, France

S. Dautheville

Emergency Department, Centre Hospitalo-Universitaire (CHU)

Tenon, Assistance-Publique Hôpitaux de Paris, Paris, France

A. Patzak

Emergency Department, Centre Hospitalo-Universitaire (CHU)

George Pompidou, Assistance-Publique Hôpitaux de Paris,

Paris, France

M. Andronikof

Emergency Department, Centre Hospitalo-Universitaire (CHU)

Antoine Béclère, Assistance-Publique Hôpitaux de Paris,

Clamart, France

\section{A. Santin}

Emergency Department, Centre Hospitalo-Universitaire (CHU) Henri Mondor, Assistance-Publique Hôpitaux de Paris,

Créteil, France

\section{S. André}

Emergency Department, Centre Hospitalo-Universitaire (CHU) Cochin-St Vincent de Paul, Assistance-Publique Hôpitaux de Paris, Paris, France

\section{Korchia}

Emergency Department, Centre Hospitalo-Universitaire (CHU)

Ambroise Paré, Assistance-Publique Hôpitaux de Paris,

Boulogne, France
N. Terbaoui

Emergency Department, Centre Hospitalo-Universitaire (CHU) Bichat-Claude Bernard, Assistance-Publique Hôpitaux de Paris, Paris, France

\section{G. Kierzek}

Emergency Department, Centre Hospitalo-Universitaire (CHU) Hôtel Dieu, Assistance-Publique Hôpitaux de Paris, Paris, France

\section{B. Doumenc}

Emergency Department, Centre Hospitalo-Universitaire (CHU)

Bicêtre, Assistance-Publique Hôpitaux de Paris, Le Kremlin

Bicêtre, France

\section{Leroy}

Emergency Department, Centre Hospitalo-Universitaire (CHU)

Louis Mourier, Assistance-Publique Hôpitaux de Paris,

Colombes, France

P. Hausfater ( $)$

Service d'Accueil des Urgences, Groupe Hospitalier Pitié-

Salpêtrière, 47-83 boulevard de l'hôpital, 75013 Paris, France e-mail: pierre.hausfater@psl.aphp.fr

Tel.: +33-1-42177249

Fax: +33-1-42177264

\section{Erratum to: Intensive Care Med DOI 10.1007/s00134-009-1694-y}

The address given for the authors P. Hausfater and B. Riou was incomplete.

Their full address is as follows:

Emergency Department, Centre Hospitalo-Universitaire (CHU) Pitié-Salpêtrière, Assistance-Publique Hôpitaux de Paris, UPMC Univ Paris 06, Paris, France. 